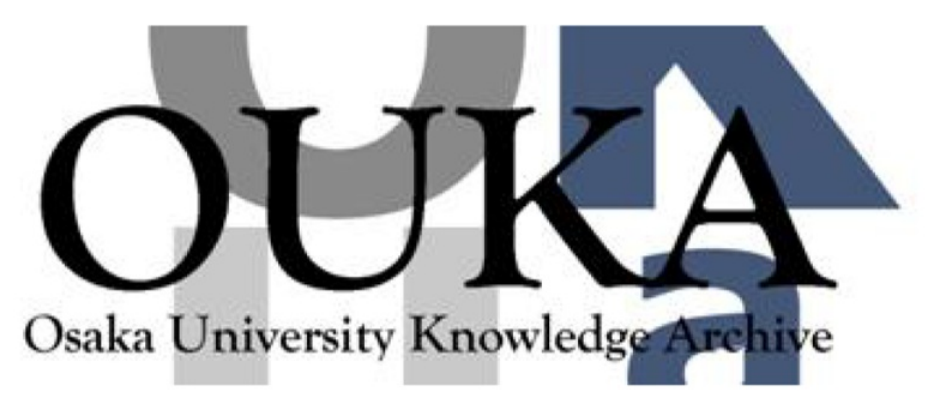

\begin{tabular}{|c|l|}
\hline Title & $\begin{array}{l}\text { Temperature tuning of the stop band in } \\
\text { transmission spectra of liquid-crystal } \\
\text { infiltrated synthetic opal as tunable photonic } \\
\text { crystal }\end{array}$ \\
\hline Author(s) & $\begin{array}{l}\text { Yoshino, Katsumi; Shimoda, Yuki; Kawagishi, } \\
\text { Yoshiaki et al. }\end{array}$ \\
\hline Citation & Applied Physics Letters. 75(7) p. 932-p.934 \\
\hline Issue Date & 1999-08-10 \\
\hline oaire:version & VoR \\
\hline URL & https://hdl. handle. net/11094/75838 \\
\hline rights & \\
\hline Note & \\
\hline
\end{tabular}

Osaka University Knowledge Archive : OUKA

https://ir. Library. osaka-u. ac. jp/

Osaka University 


\section{Temperature tuning of the stop band in transmission spectra of liquid-crystal infiltrated synthetic opal as tunable photonic crystal}

Cite as: Appl. Phys. Lett. 75, 932 (1999); https://doi.org/10.1063/1.124558

Submitted: 05 April 1999 . Accepted: 18 June 1999. Published Online: 10 August 1999

Katsumi Yoshino, Yuki Shimoda, Yoshiaki Kawagishi, Keizo Nakayama, and Masanori Ozaki

\section{ARTICLES YOU MAY BE INTERESTED IN}

Temperature effect on liquid crystal refractive indices

Journal of Applied Physics 96, 19 (2004); https://doi.org/10.1063/1.1757034

Mechanically tunable photonic crystal structure

Applied Physics Letters 85, 4845 (2004); https://doi.org/10.1063/1.1823019

Electrically color-tunable defect mode lasing in one-dimensional photonic-band-gap system containing liquid crystal

Applied Physics Letters 82, 3593 (2003); https://doi.org/10.1063/1.1577829

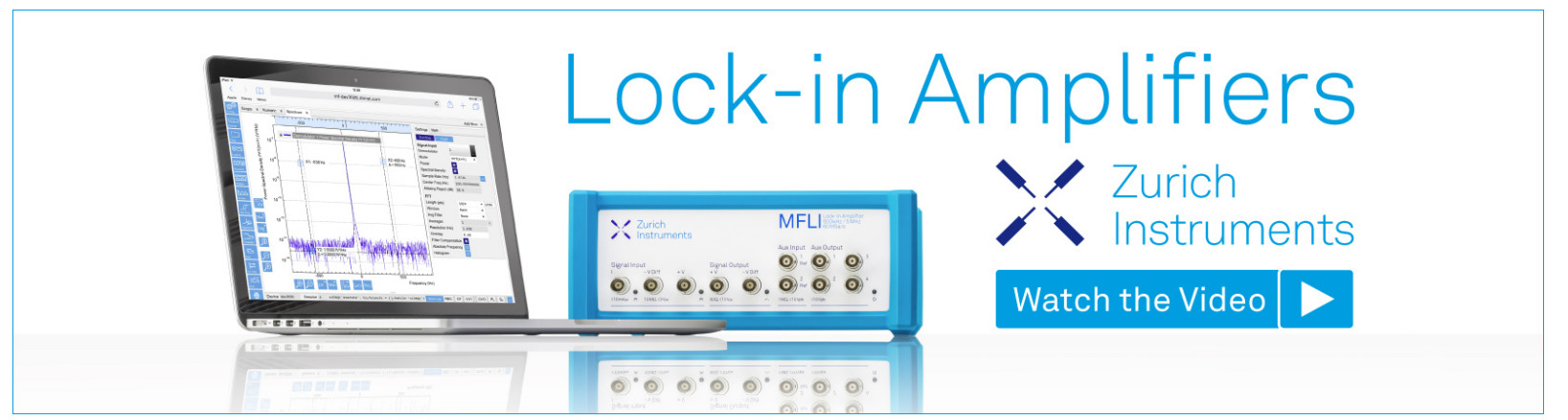




\title{
Temperature tuning of the stop band in transmission spectra of liquid-crystal infiltrated synthetic opal as tunable photonic crystal
}

\author{
Katsumi Yoshino, ${ }^{\text {a) }}$ Yuki Shimoda, Yoshiaki Kawagishi, Keizo Nakayama, and Masanori \\ Ozaki \\ Department of Electronic Engineering, Graduate School of Engineering, Osaka University, \\ 2-1 Yamada-Oka, Suita, Osaka 565-0871, Japan
}

(Received 5 April 1999; accepted for publication 18 June 1999)

\begin{abstract}
Nematic liquid crystals and smectic liquid crystals can be infiltrated into a three-dimensional periodic array of interconnected nanosize voids in opal films prepared by sedimentation of $\mathrm{SiO}_{2}$ spheres of various diameters. The optical stop band in the transmission spectra of opals shifts drastically by the infiltration of liquid crystals. The stop band is also found to shift at the phase transition points with changing temperature. This effect enables the tuning of optical properties of opals as a prototype tunable photonic crystal. This phenomenon can also be used as a measurement method for the refractive index. (C) 1999 American Institute of Physics. [S0003-6951(99)01733-7]
\end{abstract}

Recently, photonic crystals with a three-dimensional ordered structure with a periodicity of optical wavelength, have attracted much attention from both fundamental and practical points of view, because novel physical concepts such as photonic band gap have been theoretically predicted and various applications of photonic crystals have been proposed. ${ }^{1-3}$ Two types of approaches have been taken to realize the three-dimensional periodic structure, namely, some groups used microfabrication technique highly developed in a semiconductor technology. On the other hand, we have adopted a simple method utilizing sedimentation of monodispersed nanoscale spheres. This is, in some sense, a self-assembly method, and indeed the growth of natural opals from $\mathrm{SiO}_{2}$ is based on this self-assembly process.

Synthetic opals have been studied as pseudo photonic crystals or prototype photonic crystals to establish growth techniques and understand fundamental optical properties such as the stop band of the three-dimensional periodic structure of materials.

We have already demonstrated that various materials can be infiltrated into interconnected nanosize voids of opals, and novel functionalities can be realized in these infiltrated opals. ${ }^{4,5}$

We have also proposed a tunable photonic crystal in which the photonic band gap can be tuned as desired by controlling parameters such as the refractive index, periodicity, or space filling factor and also an anisotropic photonic crystal in which a three-dimensional periodic array of interconnected voids is infiltrated with anisotropic materials. ${ }^{6}$

As an initial trial for such a purpose, in this letter we demonstrate the tuning of the stop band in synthetic opals infiltrated with liquid crystals.

Ordered colloidal crystals in films were formed by sedimentation of the suspension of monodispersed silica spheres of 300 and $550 \mathrm{~nm}$ diameters in sandwich cells made of two glass [or InSn oxide (ITO) glass] plates with separations of 6,25 , and $50 \mu \mathrm{m}$. These crystals were annealed at $300^{\circ} \mathrm{C}$ for

${ }^{a}$ Electronic mail: yoshino@ele.eng.osaka-u.ac.jp about $2 \mathrm{~h}$. The typical size of the sample is $10 \times 10 \mathrm{~mm}^{2}$. In this letter, the opals made of $\mathrm{SiO}_{2}$ spheres of 300 and $550 \mathrm{~nm}$ in diameter are named as opal-300 and opal-550, respectively. Porous opals prepared by this procedure have a facecentered cubic (fcc) structure and contain interconnected structure of tetrahedral and octahedral voids. Two types of liquid crystals, nematic liquid crystal ZLI1132 (Merck) and smectic liquid crystal $(R)-4$ '-(1-methoxycarbonyl-ethoxy) phenyl-4-[4-(n-octyloxy)phenyl]benzoate (1MC1EPOPB), were used for infiltrations.

Transmission spectra through opal films were measured with a Hitachi 330 spectrophotometer. Angularly dependent transmission and reflection spectra were measured using a hand-made experimental setup with a W lamp as a light source and a PMA-11 (Hamamatsu Photonics) as a detector. Electron micrographs were taken with a S-2100 Hitachi scanning electron microscope.

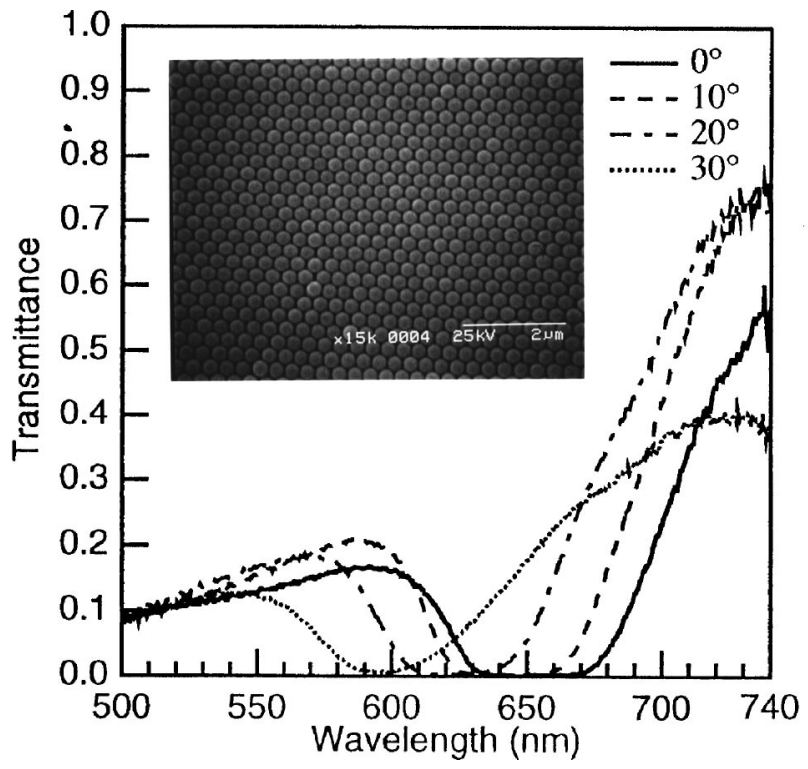

FIG. 1. Transmission spectra of opal-300 as a function of incident angle of the light. The inset is the electron micrograph of opal-300. 


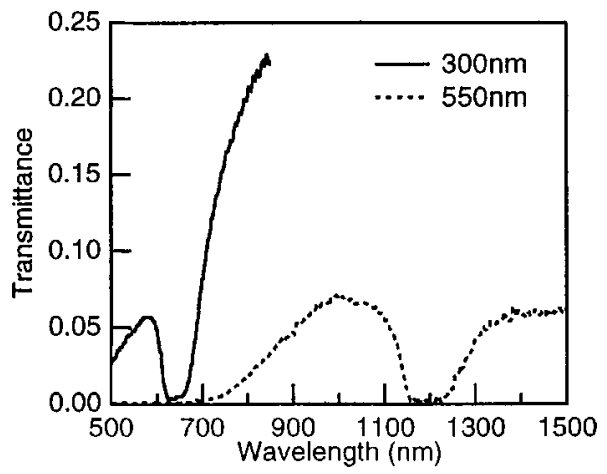

FIG. 2. Transmission spectra of opal-300 (solid line) and opal-550 (broken line) for normal incidence.

The inset of Fig. 1 shows an electron micrograph of the opal film made of $\mathrm{SiO}_{2}$ spheres of $300 \mathrm{~nm}$ in diameter. This opal film exhibits beautiful opalescent colors when irradiated with a white light and clear diffraction peaks depending on the incident angle of the light beam, which support the existence of a regular periodic nature of the obtained opal. The transmission spectra through the opal-300 thin film as a function of incident angle is shown in Fig. 1.

Figure 2 shows the transmission spectra of the opal-300 and opal-550 films for normal incidence. As is evident in this figure, clear stop bands appear at the wavelengths of around 640 and $1200 \mathrm{~nm}$ in the opal-300 and opal-550, respectively. The diameters of $\mathrm{SiO}_{2}$ spheres of the opal-300 and opal-550, evaluated from the observed diffraction and transmission spectra by assuming fcc lattice, coincide nearly with those of the original spheres.

Figure 3 indicates transmission spectra of the opal-300 and the opal-300 infiltrated with nematic liquid crystal (ZLI1132). As is evident in this figure, the stop band of the nematic liquid crystal infiltrated opal appears at longer wavelength by about $70 \mathrm{~nm}$ in comparison with that of the opal300 itself, which should originate from the large refractive index of ZLI1132. Similar redshift of the stop band was also observed in the nematic liquid crystal infiltrated opal-550, that is, at $1340 \mathrm{~nm}$ in the nematic liquid crystal infiltrated opal-550 and at $1200 \mathrm{~nm}$ in the opal-550. It should also be noted in the inset of Fig. 3 that even in the same nematic

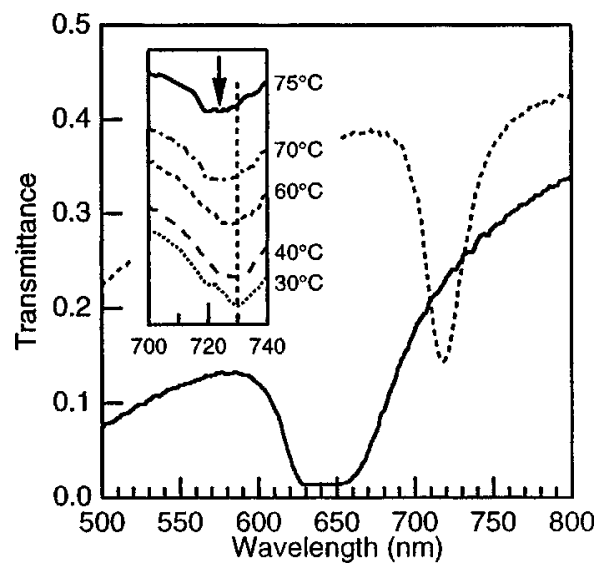

FIG. 3. Transmission spectra of opal-300 (solid line) and opal-300 infiltrated with nematic liquid crystal (broken line). The inset shows the temperature dependence of transmission spectra.

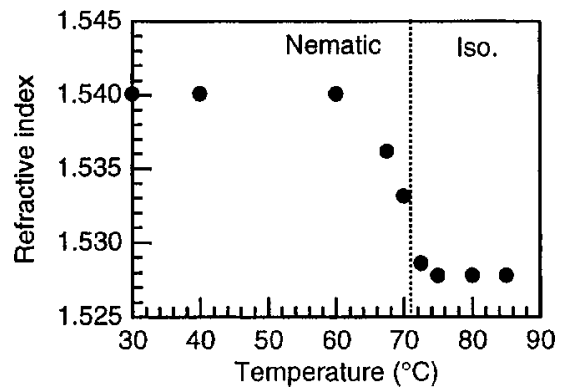

FIG. 4. Temperature dependence of evaluated refractive index of nematic liquid crystal (ZLI1132) in voids of opal-300.

liquid crystal infiltrated opal, the stop band shifts with changing temperature. From these spectral change, we can evaluate the refractive index of the liquid crystal by utilizing the periodicity of the opal and the refractive index of $\mathrm{SiO}_{2}$ as a function of temperature.

Figure 4 shows the temperature dependence of the refractive index of ZLI1132. With decreasing temperature, stepwise increase of the refractive index was observed at the phase transition point between the isotropic and nematic phases. The refractive index in the nematic phase should be an averaged value of the refractive indices for the ordinary and extraordinary lights. The magnitude of the step of the refractive index was about 0.012, which is comparable to that evaluated by the ordinary measurement method of the refractive index.

The stop band of the opal infiltrated with ferroelectric liquid crystal 1MC1EPOPB also exhibited remarkable red shift as shown in Fig. 5. It should also be noted in the inset of this figure that the stop band of $1 \mathrm{MC} 1 \mathrm{EPOPB}$ infiltrated opal-300 shifts with depending on the liquid crystal phases.

From the temperature dependence of the stop band wavelength, the refractive index of $1 \mathrm{MC} 1 \mathrm{EPOPB}$ is evaluated as shown in Fig. 6. In this figure, also the stepwise changes of the refractive index were observed at phase transition points.

We are now studying the dependence of the stop band of liquid crystal infiltrated opals on applied field.

In summary, we demonstrated that the stop band of the

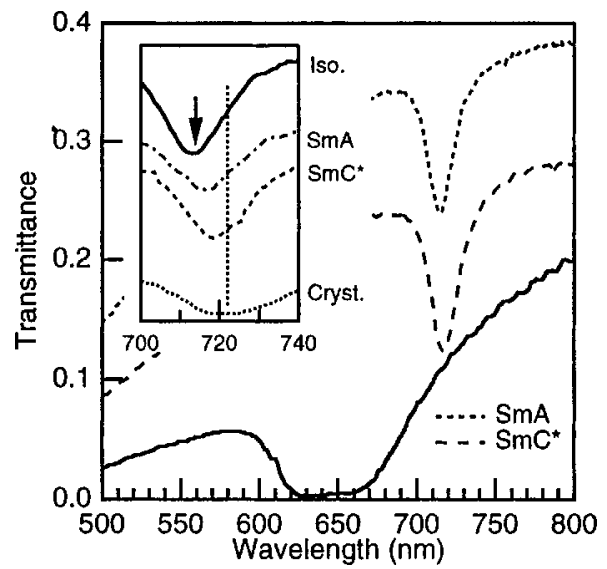

FIG. 5. Transmission spectra of opal-300 (solid line) and opal-300 infiltrated with ferroelectric liquid crystal showing the smectic A and chiral smectic C phases (broken lines). The inset shows the temperature dependence of transmission spectra. 


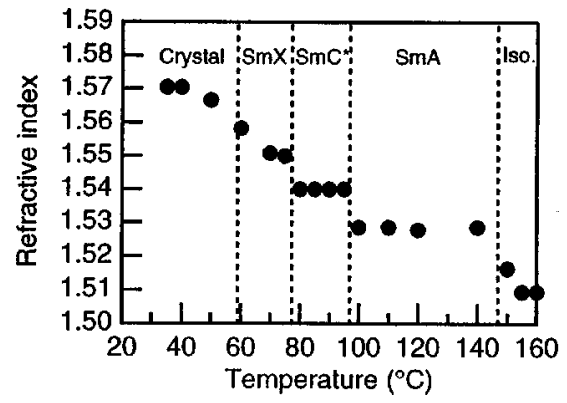

FIG. 6. Temperature dependence of evaluated refractive index of ferroelectric liquid crystal (1MC1EPOPB) in voids of opal-300.

opal can be clearly tuned by infiltrating liquid crystals and changing the temperature, which seems to support the possibility of the tunable photonic crystals. It should also be mentioned that we demonstrated a measurement method for the refractive index. That is, by observing the transmission spectra through infiltrated opals, the refractive index of the infiltrated material can be evaluated.

This work was supported by NEDO International Joint Research Grant.

${ }^{1}$ S. John, Phys. Rev. Lett. 58, 2486 (1987).

${ }^{2}$ E. Yablonovitch, Phys. Rev. Lett. 58, 2059 (1987).

${ }^{3}$ S. John and T. Quang, Phys. Rev. Lett. 74, 3419 (1995).

${ }^{4}$ K. Yoshino, K. Tada, M. Ozaki, A. A. Zakhidov, and R. H. Baughman, Jpn. J. Appl. Phys., Part 2 36, L714 (1997).

${ }^{5}$ K. Yoshino, S. Tatsuhara, Y. Kawagishi, M. Ozaki, A. A. Zakhidov, and Z. V. Vardeny, Appl. Phys. Lett. 74, 2590 (1999).

${ }^{6}$ K. Yoshino, Production Technique 50, 26 (1998) (in Japanese). 